\title{
Predictability of Optical Coherence Tomography Parameters in Preperimetric Glaucoma
}

\section{Dalia Sabry ${ }^{1}$, Ahmed S Elwehidy ${ }^{1}$, Ameera Gamal Abdelhameed ${ }^{2}$, Ehab Tharwat $^{3}$ and Amr M Abdelkader ${ }^{2 *}$}

${ }^{1}$ Professor of Ophthalmology, Faculty of Medicine, Mansoura University, Mansoura,

Egypt

${ }^{2}$ Lecturer of Ophthalmology, Faculty of Medicine, Mansoura University, Mansoura,

Egypt

${ }^{3}$ Lecturer of Ophthalmology, Faculty of Medicine, Al-Azhar University, Damietta,

Egypt

*Corresponding Author: Amr M Abdelkader, Lecturer of Ophthalmology, Faculty of Medicine, Mansoura University, Mansoura, Egypt.
Received: January 08, 2021

Published: February 16, 2021

(C) All rights are reserved by Amr $\mathbf{M}$

Abdelkader., et al.

\begin{abstract}
Purpose: To investigate the diagnostic value of inner macular layers (IMLs) thickness, Bruch's membrane opening-minimum rim width (BMO-MRW) and peripapillary retinal nerve fiber layer thickness (pRNFLT) in the diagnosis of pre perimetric glaucoma (PPG).

Patients and Methods: This was an observational study included 79 eyes with PPG and 58 control eyes. Measurements of the BMOMRW, pRNFLT and individual IMLs including the macular retinal nerve fiber layer (mRNFL), ganglion cell layer (GCL) and inner plexiform layer (IPL) were performed using Premium glaucoma Module for Spectralis SD-OCT.

Results: PPG parameters showed a statistically significant difference with control eyes except the superonasal sector of the pRNFLT. Apart from superonasal sector of the pRNFLT, Spearman correlation showed a positive correlation between PPG and all tested parameters. GCL and IPL, followed by the global pRNFL had the strongest predictability of PPG as proved by binary logistic regression analysis.

Conclusion: Individual IMLs (mRNFL, GCL and IPL), pRNFL and BMO-MRW and their subsectors were helpful in detecting PPG. GCL and IPL showed the highest predictability followed by the global thickness of the pRNFLT.
\end{abstract}

Keywords: Glaucoma; OCT; IMLs; BMO-MRW; pRNFL; mRNFL; GCL; IPL

\section{Abbreviations}

IMLs: Inner Macular Layers; BMO-MRW: Bruch's Membrane Opening-minimum Rim Width; pRNFLT: Peripapillary Retinal Nerve Fiber Layer Thickness; PPG: Pre Perimetric Glaucoma; mRNFL: Macular Retinal Nerve Fiber Layer; GCL: Ganglion Cell Layer; IPL: Inner Plexiform Layer

\section{Introduction}

Glaucoma is a progressive optic neuropathy with loss of retinal ganglion cells and their axons. This results in thinning of the neuroretinal rim of the optic nerve head $(\mathrm{ONH})$ and retinal nerve fiber layer (RNFL) together with visual field (VF) loss [1,2]. Significant structural changes occur prior to the development of functional VF loss [3]. Detection of early structural changes is fundamental 
to early diagnosis of PPG [4]. The peripapillary RNFL thickness (pRNFLT) is the traditional element used for early detection of glaucoma. However, recently, the Bruch's membrane openingminimum rim width (BMO-MRW), has been suggested as a valid alternative structural measure with better diagnostic accuracy in PPG than traditional parameters like pRNFLT [5-9]. The BMO-MRW is the minimum distance between the BMO and the surface of the internal limiting membrane (ILM) [6]. A stronger association of VF sensitivity has also been found with BMO-MRW than other $\mathrm{ONH}$ parameters or pRNFLT [9]. Macular damage in early glaucoma is recognized as a common disease feature that have negative impact on vision-related quality of life (VRQoL) [10-15]. Spectralis OCT have allowed segmental analysis of IMLs thickness; macular nerve fiber layer (mRNFL), ganglion cell layer (GCL) and inner plexiform layer (IPL) [16]. It was suggested that measurement of IMLs thickness is better than pRNFLT to detect early glaucoma, because the IMLs are involved at an earlier stage. Furthermore, the variation in GCL thickness measurements is lower than that of pRNFLT and $\mathrm{ONH}$ parameters because disc tilting and torsion are common $[17,18]$. The aim of this study was to investigate the diagnostic value of BMO-MRW, pRNFLT and individual IMLs thickness in the diagnosis of PPG.

\section{Patients and Methods}

This observational study was performed on patients with PPG and a control group of age-matched normal subjects. Participants were enrolled at the Investigative Unit of Mansoura Ophthalmic Center, Mansoura, Egypt, in the period between February and September 2019. The study was performed in accordance with the tenets of the declaration of Helsinki and was approved by the institutional review board (IRB) of Mansoura University. Study participants provided written informed consent after explanation of the procedure of the study. Pre-perimetric glaucoma (PPG) was defined as focal or diffuse RNFL defects on infra-red images corresponding to glaucomatous optic disc changes and a normal VF test [29]. Glaucomatous optic disc changes were defined as $>0.7$ vertical cup to disc ratio (C/D), > 0.2 asymmetric C/D between the glaucomatous and normal eyes and neuroretinal rim thinning, notching, or excavation on optic disc photography [29]. The IOP of patients with PPG included in the study was controlled with antiglaucoma medications. Control subjects were defined as those with no history of ocular disease, absence of glaucomatous optic disc appearance, IOP less than $21 \mathrm{mmHg}$, and a normal VF. All enrolled subjects underwent a complete ophthalmic examination. Examination included measurement of best-corrected visual acuity (BCVA), IOP measurement by Goldmann applanation tonometry, non-cycloplegic refraction, Slit-lamp biomicroscopy, gonioscopy, fundoscopy, and central corneal thickness measurement (CCT) using ultrasonic pachymeter. Exclusion criteria included a spherical equivalent (SE) more than -6 diopters (D) to $+2 \mathrm{D}$ (in order not to avoid the effect of high refractive errors on OCT measurements), closed or abnormal anterior chamber angle on gonioscopy, diabetes, uveitis, secondary glaucoma, corneal abnormalities, nonglaucomatous optic neuropathies, previous trauma, ocular surgery or laser treatment, and/or abnormal disk appearance such as tilted disk, non-glaucomatous disk damage, or extensive peripapillary atrophy. Swedish interactive threshold algorithm (SITA) standard strategy, program 24-2 of the Humphrey Field Analyzer (Carl Zeiss Meditec, Jena, Germany), was used for VF testing of each eye. VF testing was repeated for each subject. The first test was considered an educational one to enhance participant's performance. VF testing and OCT scanning were performed in a separate day to eliminate the effect of pupillary dilatation when examining the fundus clinically. The study was designed to investigate the diagnostic value of BMO-MRW, pRNFLT, mRNFL, GCL and IPLs thickness in the diagnosis of PPG using Premium glaucoma Module for Spectralis SD-OCT (Software Version 6.01; Heidelberg Engineering, Germany). A single experienced operator (DS) performed OCT examination. No pupillary dilatation was needed. A $30^{\circ}$ macular line scan was performed first to make the participants familiar with the internal fixation target. A wellfocused infra-red image with visible peripapillary retinal nerve fiber layer ( $\mathrm{pRNFL}$ ) striation was obtained before glaucoma module is activated by selecting the infra-red image mode and changing the site of the fixation target nasally to make the optic disc at the image center. Then the glaucoma module was activated. We started examination with Optic Nerve Head-Radial Circle (ONH-RC) scan that combines 24 radial scans and three concentric circle scans centered on the ONH with anatomic position system (APS). The APS starts automatically and uses the TruTrack image alignment software with an automatic real-time function. Using this system, multiple frames of exactly the same locations can be performed during the scanning process. It creates an anatomic map for each participant's eye using two fixed anatomical landmarks: the center of the fovea and the center of the BMO (FoBMO axis). The software starts foveal detection with a live two line B-scans perpendicular 
to each other. The detected foveal center should be confirmed with the operator. In a similar fashion, it detects the center of BMO. Then the individual APS map is created by a line joining the foveal center with the center of the BMO. After acquiring the participant's APS a 24 high resolution radial B-scans (15 degrees apart), centered on the BMO is automatically activated. It measures the neuroretinal rim parameters. Automatically computed globally and sectorally (superotemporal, superonasal, nasal, inferonasal, inferotemporal, and temporal sector) measurement of the MRW is acquired. The ONH-RC then provides three peripapillary circle scans with diameters of: $3.5 \mathrm{~mm} 4.1 \mathrm{~mm}$ and $4.7 \mathrm{~mm}$. All circle scans are aligned to the individual FoBMO axis. The alignment ensures an accurate definition of each single sector (superotemporal, superonasal, nasal, inferonasal, inferotemporal, and temporal sector) independent of head positions and thereby enables a correct comparison to the reference database. The $3.5 \mathrm{~mm}$ circle diameter is the standard one from which the global and sector pRNFLT is obtained. The additional two larger circle scan diameters are adopted in cases when the inner circle is not interpretable due to an influencing pathology as peripapillary cotton wool spots or myelinated nerve fibers. The posterior pole measurements are obtained by the posterior pole horizontal scan (P PoleH). The PPoleH scan is a volume scan which is placed on the posterior pole of the eye aligned to the individual FoBMO axis. It consists of 61 horizontal B-scans $120 \mu \mathrm{m}$ apart with dimensions of $30 \times 25$ degrees. The axial resolution is $7 \mu \mathrm{m}$ and the lateral resolution is $14 \mu \mathrm{m}$. None of the included eyes needed manual readjustment of the arrow between the BMO and nearest point of the ILM. Then the BMO-MRW thickness curve was reviewed to detect sites of notching and confirm that they are due to actual thinning and not due to any artifacts. Also the curve was reviewed for any abnormal thickening or thinning. The segmentation of the 3.5 $\mathrm{mm}$ circle scan was revised by the examiner for any abnormities (i.e. the ILM or the RNFL lines are in inaccurate position). posterior pole assessment was achieved by clicking the PPoleH scan segment all layers button. Then it was opened where a 64-box grid appeared overlying the central $24^{\circ} \times 24^{\circ}$ of the macular region. Each box of the grid is $3^{\circ} \times 3^{\circ}$ and represents the average macular thickness in the region that it overlies. To make sure of the accurate segmentation and centration of the measurement the grid was moved manually if necessary so that its center coincide with the foveal center and was rotated so that its central line coincide with the Fo-BMO axis. The Spectralis automatically segmented the B scans from the inner limiting membrane to Bruch membrane. None of PPG or control sub- jects showed any segmentation errors. The glaucoma module print out was ordered from which we obtained the BMO-MRW and pRNFLT in the nasal, temporal superonasal, superotemporal, inferonasal, inferotemporal and global thickness. Also from the macular hemisphere asymmetry square in the same print out we obtained the total, average superior and average inferior macular thickness. Then the posterior pole hemisphere asymmetry printout for each mRNFL, GCL and IPL were ordered from which we obtained the total, average superior and average inferior thickness.

\section{Statistical analysis}

Data were coded, computed then analyzed using IBM SPSS (Statistical package for social science) version 24 for Windows. Descriptive statistics were calculated in the form of mean \pm SD. P value less than 0.05 (5\%) was considered to be statistically significant. MannWhitney test was used to compare different parameters among the glaucomatous and the non-glaucomatous control group. Bivariate correlation using "Spearman correlation" was used to explore the significant correlation between different imaging parameters and the possibility of coexistence of PPG. Binary logistic regression analysis was used to compare the predictability of non-glaucoma (normality) for each parameter "Odd's ratio" codding "0" for glaucomatous group and " 1 " for normality. So the statistical model was designed to predict the probability "odd's ratio" of normality.

\section{Results}

The current study was an observational study, including 79 eyes with PPG and 58 control eyes. There were no significant differences between groups in sex, age, spherical equivalence (SE), CCT, VF mean deviation (MD) or pattern standard deviation (PSD). However, PPG eyes showed significantly higher IOP compared to controls. The demographic and clinical characteristics of the subjects are included in table 1. A statistically significant difference between PPG cohort and control group was obtained in the total, superior and inferior thickness of the GCL, IPL and mRNFL ( $\mathrm{P}<0.0001)$, Also the total, superior and inferior retinal thickness showed statistically significant difference between PPG cohort and control group that was most evident inferiorly. The BMO-MRW global value and each of its subsectors "inferonasal, inferotemporal, nasal, temporal, superonasal and superotemporal" did achieve a statistically significant difference between the PPG and control groups $(\mathrm{P}<0.0001)$. The traditionally used parameter pRNFLT also showed a significant difference $(\mathrm{P}<0.0001)$ in its global and subsectors thickness ex- 
cept the superonasal sector that showed insignificant difference $(\mathrm{P}$ $=0.134$ ). The significance difference between control and PPG subjects are shown in table 2. Correlation between different parameters and the possibility of existence of PPG were highly significant in GCL, IPL and mRNFL, as a total value together with their superior and inferior subsectors. The total, superior and inferior average retinal thickness was significantly correlated with PPG most evident in the inferior half. These correlations were maintained with the "BMO-MRW thickness" and its subsectors. The traditional parameter pRNFLT and its subtypes showed positive correlation except in its superonasal sector. Bivariate correlations between different parameters and PPG group are shown in table 3. GCL and its subsectors appeared to have the maximum predictability of PPG. The next predictor factor of PPG was found to be IPL and its superior and inferior subsectors. The mRNFL as a total value and each of its subsectors expressed less evident but still good predictability of the PPG. The total average retinal thickness had no significant predictability of the PPG; however its superior and inferior retinal thickness showed some predictability effect. The BMO-MRW thickness was found to have an inferior predictability effect of PPG compared with the traditional parameter "pRNFLT" in their global values. Although the thickness of all MRW subsectors apart from the superonasal sector had a statistically significant predictability effect with PPG. On the other hand, all pRNFL subsectors except the superior and inferior temporal sectors had poor significant predictability values of PPG. Logistic regression analysis (Predicted Probability of membership for PPG) is shown in table 4.

\begin{tabular}{|l|c|c|c|}
\hline & Normal (58) & PPG (79) & P Value \\
\hline Age & $41,79 \pm 15.79$ & $40.27 \pm 19.01$ & 0.672 \\
\hline Male/Female & $10 / 19$ & $19 / 24$ & 0.466 \\
\hline SE (D) & $-1.75 \pm 0.41$ & $-1.72 \pm 0.62$ & 0.326 \\
\hline IOP $(\mathrm{mmHg})$ & $16.43 \pm 0.24$ & $18.15 \pm 0.58$ & 0.001 \\
\hline CCT $(\mu \mathrm{m})$ & $550 \pm 13$ & $547 \pm 9$ & 0.137 \\
\hline MD $(\mathrm{dB})$ & $-1.38 \pm 0.47$ & $-1.49 \pm 0.72$ & 0.198 \\
\hline PSD $(\mathrm{dB})$ & $1.11 \pm 0.93$ & $1.02 .4 \pm 1.31$ & 0.147 \\
\hline
\end{tabular}

Table 1: The demographic and clinical characteristics of the subjects.

$\mathrm{SE}=$ Spherical Equivalent; $\mathrm{D}=$ Diopter; $\mathrm{IOP}=$ Intraocular Pressure; $\mathrm{mmHg}=$ Millimeter Mercury; $\mathrm{CCT}=$ Central Corneal Thickness; $\mu \mathrm{m}=$ Micron; $\mathrm{MD}=$ Mean Deviation; $\mathrm{dB}=$ Decibel; $\mathrm{PSD}=$ Pattern Deviation.

${ }^{*} \mathrm{P}<0.05$ is considered as statically significant.

\begin{tabular}{|c|c|c|c|}
\hline Thickness & $\begin{array}{c}\text { Control } \\
\text { Mean } \pm \text { SD } \\
(\mu \mathrm{m})\end{array}$ & $\begin{array}{c}\text { PPG } \\
\text { Mean } \pm \text { SD } \\
(\mu \mathrm{m})\end{array}$ & $P$ value \\
\hline \multicolumn{4}{|l|}{ pRNFL } \\
\hline Global & $116.38 \pm 27.66$ & $96.11 \pm 10.01$ & $<0.0001$ \\
\hline Nasal & $94.43 \pm 13.327$ & $83.4 \pm 12.78$ & $<0.001$ \\
\hline Superonasal & $128.29 \pm 20.24$ & $121.69 \pm 24.92$ & 0.134 \\
\hline Inferonasal & $134.80 \pm 21.28$ & $110.58 \pm 22.29$ & $<0.0001$ \\
\hline Temporal & $77.35 \pm 10.58$ & $67.78 \pm 9.82$ & $<0.0001$ \\
\hline $\begin{array}{l}\text { Superotem- } \\
\text { poral }\end{array}$ & $149.38 \pm 19.66$ & $115.07 \pm 20.64$ & $<0.0001$ \\
\hline Inferotemporal & $168.52 \pm 22.15$ & $135.8 \pm 24.51$ & $<0.0001$ \\
\hline \multicolumn{4}{|l|}{ BMO-MRW } \\
\hline Global & $346.08 \pm 49.48$ & $273.26 \pm 48.51$ & $<0.0001$ \\
\hline Nasal & $374.21 \pm 65.70$ & $282.77 \pm 71.61$ & $<0.0001$ \\
\hline Superonasal & $391.26 \pm 61.27$ & $298.82 \pm 62.89$ & $<0.0001$ \\
\hline Inferonasal & $424.40 \pm 77.83$ & $322.03 \pm 73.43$ & $<0.0001$ \\
\hline Temporal & $240.05 \pm 43.71$ & $205.00 \pm 53.01$ & $<0.0001$ \\
\hline $\begin{array}{l}\text { Superotem- } \\
\text { poral }\end{array}$ & $339.82 \pm 52.67$ & $260.07 \pm 56.04$ & $<0.0001$ \\
\hline Inferotemporal & $377.07 \pm 62.46$ & $298.45 \pm 63.36$ & $<0.0001$ \\
\hline \multicolumn{4}{|l|}{ Average macular } \\
\hline Total & $289.12 \pm 19.28$ & $282.87 \pm 14.48$ & 0.002 \\
\hline Superior & $291.21 \pm 12.65$ & $\begin{array}{c}283.11 \pm \\
14.039 \\
\end{array}$ & 0.001 \\
\hline Inferior & $292.36 \pm 15.51$ & $282.58 \pm 15.11$ & $<0.001$ \\
\hline \multicolumn{4}{|l|}{ mRNFL } \\
\hline Total & $41.48 \pm 5.11$ & $35.68 \pm 5.04$ & $<0.0001$ \\
\hline Superior & $38.36 \pm 4.48$ & $32.79 \pm 4.81$ & $<0.0001$ \\
\hline Inferior & $44.48 \pm 6.32$ & $38.45 \pm 6.28$ & $<0.0001$ \\
\hline \multicolumn{4}{|l|}{ GCL } \\
\hline Total & $34.59 \mu \pm 2.67$ & $31.13 \pm 3.24$ & $<0.0001$ \\
\hline Superior & $34.65 \pm 2.69$ & $31.01 \pm 3.37$ & $<0.0001$ \\
\hline Inferior & $34.61 \pm 3.01$ & $31.41 \pm 3.34$ & $<0.0001$ \\
\hline \multicolumn{4}{|l|}{ IPL } \\
\hline Total & $28.67 \pm 2.24$ & $26.62 \pm 2.49$ & $<0.0001$ \\
\hline Superior & $29.01 \pm 2.25$ & $26.77 \pm 2.57$ & $<0.0001$ \\
\hline Inferior & $28.33 \pm 2.35$ & $26.41 \pm 2.48$ & $<0.0001$ \\
\hline
\end{tabular}

Table 2: Significance difference between control and pre perimetric glaucoma subjects

$\mathrm{PPG}=$ Pre Perimetric Glaucoma, pRNFL= Peripapillary Retinal Nerve Fiber Layer; BMO-MRW = Bruch's Membrane Opening Minimum Rim Width; mRNFL= Macular Retinal Nerve Fiber Layer; GCL= Ganglion Cell Layer; IPL= Inner Plexiform Layer.

$* \mathrm{P}<0.05$ is considered as statistically significant. 


\begin{tabular}{|c|c|c|}
\hline Thickness & $\begin{array}{c}\text { Spearman } \\
\text { Correlation with } \\
\text { PPG }\end{array}$ & $\begin{array}{c}\text { Sig. } \\
\text { (2-tailed) }\end{array}$ \\
\hline \multicolumn{3}{|l|}{ pRNFL } \\
\hline Global & 0.460 & $<0.0001$ \\
\hline Nasal & 0.378 & $<0.0001$ \\
\hline Superonasal & 0.141 & 0.102 \\
\hline Inferonasal & 0.482 & $<0.0001$ \\
\hline Temporal & 0.424 & $<0.0001$ \\
\hline Superotemporal & 0.644 & $<0.0001$ \\
\hline Inferotemporal & 0.571 & $<0.0001$ \\
\hline \multicolumn{3}{|l|}{ BMO-MRW } \\
\hline Global & 0.595 & $<0.001$ \\
\hline Nasal & 0.549 & $<0.001$ \\
\hline Superonasal & 0.594 & $<0.001$ \\
\hline Inferonasal & 0.560 & $<0.001$ \\
\hline Temporal & 0.333 & $<0.001$ \\
\hline Superotemporal & 0.587 & $<0.001$ \\
\hline Inferotemporal & 0.527 & $<0.001$ \\
\hline \multicolumn{3}{|l|}{ Average Macular } \\
\hline Total & 0.183 & 0.033 \\
\hline Superior & 0.282 & 0.001 \\
\hline Inferior & 0.303 & 0.0001 \\
\hline \multicolumn{3}{|l|}{ mRNFL } \\
\hline Total & 0.492 & $<0.0001$ \\
\hline Superior & 0.507 & $<0.0001$ \\
\hline Inferior & 0.428 & $<0.0001$ \\
\hline \multicolumn{3}{|l|}{ GCL } \\
\hline Total & 0.484 & $<0.0001$ \\
\hline Superior & 0.485 & $<0.0001$ \\
\hline Inferior & 0.439 & $<0.0001$ \\
\hline \multicolumn{3}{|l|}{ IPL } \\
\hline Total & 0.392 & $<0.0001$ \\
\hline Superior & 0.414 & $<0.0001$ \\
\hline Inferior & 0.365 & $<0.0001$ \\
\hline
\end{tabular}

Table 3: Bivariate correlations between different parameters and pre perimetric glaucoma group.

pRNFL= Peripapillary Retinal Nerve Fiber Layer; BMO-MRW = Bruch's Membrane Opening Minimum Rim Width; $\mathrm{mRNFL=}$ Macular Retinal Nerve Fiber Layer; GCL= Ganglion Cell Layer; IPL= Inner Plexiform Layer; PPG= Pre Perimetric Glaucoma.

\begin{tabular}{|c|c|c|c|}
\hline Thickness & $\begin{array}{l}\text { Odds } \\
\text { ratio }\end{array}$ & $\mathbf{P}$ & $\begin{array}{c}\text { Confidence } \\
\text { interval }\end{array}$ \\
\hline \multicolumn{4}{|l|}{ pRNFL } \\
\hline Global & 1.202 & $<0.001$ & $1.129-1.279$ \\
\hline Nasal & 1.02 & 0.28 & $0.97-1.07$ \\
\hline Superonasal & 0.98 & 0.33 & $0.96-1.01$ \\
\hline Inferonasal & 1.00 & 0.56 & $0.97-1.03$ \\
\hline Temporal & 1.00 & 0.92 & $0.94-1.06$ \\
\hline Superotemporal & 1.09 & $<0.001$ & $1.04-1.13$ \\
\hline Inferotemporal & 1.08 & $<0.001$ & $1.04-1.12$ \\
\hline \multicolumn{4}{|l|}{ BMO-MRW } \\
\hline Global & 1.03 & $<0.0001$ & $1.02-1.04$ \\
\hline Nasal & 1.07 & $<0.0001$ & $1.035-1.06$ \\
\hline Superonasal & 1.013 & $<0.104$ & 0.997-1.028 \\
\hline Inferonasal & 1.052 & $<0.0001$ & $1.031-1.073$ \\
\hline Temporal & 1.094 & $<0.0001$ & $1.053-1.138$ \\
\hline Superotemporal & 1.09 & $<0.0001$ & 1.059-1.123 \\
\hline Inferotemporal & 1.072 & $<0.0001$ & $1.046-1.098$ \\
\hline \multicolumn{4}{|l|}{ Average Macular } \\
\hline Total & 1.01 & 0.35 & $0.98-1.03$ \\
\hline Superior & 1.02 & $<0.001$ & $1.00-1.05$ \\
\hline Inferior & 1.03 & $<0.001$ & $1.00-1.06$ \\
\hline \multicolumn{4}{|l|}{ mRNFL } \\
\hline Total & 1.026 & $<0.036$ & $1.002-1.05$ \\
\hline Superior & 1.045 & 0.02 & $1.05-1.07$ \\
\hline Inferior & 1.044 & $<0.001$ & $1.02-1.07$ \\
\hline \multicolumn{4}{|l|}{ GCL } \\
\hline Total & 1.496 & $<0.0001$ & $1.27-1.76$ \\
\hline Superior & 1.482 & $<0.0001$ & $1.26-1.74$ \\
\hline Inferior & 1.399 & $<0.0001$ & $1.21-1.62$ \\
\hline \multicolumn{4}{|l|}{ IPL } \\
\hline Total & 1.43 & $<0.0001$ & $1.21-1.69$ \\
\hline Superior & 1.45 & $<0.0001$ & $1.23-1.71$ \\
\hline Inferior & 1.39 & $<0.0001$ & $1.19-1.64$ \\
\hline
\end{tabular}

Table 4: Logistic regression analysis (Predicted Probability of membership for pre perimetric glaucoma).

pRNFL= Peripapillary Retinal Nerve Fiber Layer; BMO-MRW = Bruch's Membrane Opening Minimum Rim Width; mRNFL= Macular Retinal Nerve Fiber Layer; GCL= Ganglion Cell Layer; IPL= Inner Plexiform Layer. Confidence interface 95\% C.I.for EXP (B). 


\section{Discussion}

This study was designed to evaluate diagnostic value of the newly introduced BMO-MRW and IMLs versus traditional pRNFLT in early detection of PPG. Although all these parameters and most of their subsectors were statistically affected in PPG, the strongest association and predictability of PPG was marked with IMLs and global pRNFLT. This study was designed because macular damage in early glaucoma was reported in several studies and their authors suggested investigating the validity of macular imaging for monitoring patients in early and established glaucoma [12,19-21]. The current study together with a recent group of studies confirmed the diagnostic value of macular imaging in early detection and follow up of glaucoma [22-26]. Chen., et al. investigated the diagnostic ability of macular ganglion cell asymmetry in PPG and found that asymmetrical measurements of ganglion cell inner plexiform layer (GCIPL) have diagnostic ability comparable to pRNFL, and $\mathrm{ONH}$ analysis for PPG [19]. However their study was limited by the use of Cirrus HD-OCT macular cube that covers only the foveal and parafoveal regions and provides GCIPL complex thickness instead of individual GCL thickness. This could be attributed to the lack of adequate segmentation by most available commercial OCT devices and lack of normative database. With the advances of OCT technology, using the posterior pole asymmetry protocol of Spectralis we were able to obtain accurate and automated segmentation of the individual IMLs (mRNFL, GCL and IPL) and to obtain their thickness measurements all-over the entire macula with $24^{\circ} \times 24^{\circ}$ square of measurement. Additionally, this protocol allows accurate centration of the scan on the macular center and on the FoBMO axis that with the Tru-Track image alignment software, enabled reproducible scans and accurate follow up. In our study we were able not only to detect IML thinning but also to show that the GCL thickness changes followed by the IPL carried the highest predictability for PPG. Our findings suggest that glaucomatous damage starts in the inner macular layers specially the GCL. The findings suggest the importance of follow up of these layers using the patient's baseline evaluation at the present time to detect early progression before established VF changes appears. It is advisable to change the current recommendations and preferred practice patterns (PPP) to include IMLs (mRNFL, GCL and IPL) with pRNFLT and $\mathrm{ONH}$ parameters in screening and monitoring early glaucoma progression. In our study the total average retinal thickness had no significant predictability of the PPG. However its superior and inferior sectors showed some predictability effect. This suggests that the standard macular thickness with the 9 ETDRS regions is not recommended for PPG assessment in contrast to the individual IMLs specially GCL and IPL. Our findings In BMO-MRW and pRNFLT are in agreement with Gmeiner., et al. who performed comparison of BMO-MRW and pRNFLT in early glaucoma assessment [9]. Their findings were consistent with our results that global pRNFLT yielded higher performance in distinguishing PPG. They reported variable diagnostic performance among different sectors of pRNFLT with two peaks in superior and inferior analyses. The reported pattern was evident in the pRNFLT and not in the BMO-MRW. Their explanation was based on the anatomical distribution of pRNFLT, which features thicker regions in inferior and superior sectors, whereas BMO-MRW is more evenly arranged. And they speculated that BMO-MRWs performance may be more consistent throughout all sectors. These findings were confirmed by other studies that reported the two peaks of pRNFLT affection rather than even affection $[27,28]$. This high predictability of the global pRNFLT and its specific subsectors affection keeps it an important element in the diagnosis of PPG. Park., et al. investigated the reproducibility of BMO-MRW measurements with glaucoma module of Spectralis OCT [29].They reported excellent reproducibility in BMO-MRW measurements in both normal and glaucoma subjects. In our study we used the same device and its module. We found that BMO-MRW exhibited good predictability for PPG in its global and subsector thickness apart from the superonasal sector. However we did not find a specific pattern for early glaucoma detection as that found in the superotemporal and inferotemporal sectors of the pRNFL. Detecting early glaucoma and monitoring glaucomatous progression are different tasks. Therefore it is not realistic to assume that the same parameters or analyses will be optimal for both. With excellent reproducibility of the measurement of the BMO-MRW and even affection of its sectors it may be more appropriate to use it for follow up of the disease progression.

\section{Conclusion}

Measuring multiple parameters including the individual IMLs (mRNFL, GCL and IPL), pRNFL and BMO-MRW improves the ability to diagnose PPG. GCL and IPL showed the highest predictability for PPG followed by the global thickness of the pRNFLT.

\section{Conflict of Interest}

The authors have no financial interest in any of the material stated in the study. 


\section{Acknowledgements}

This study is an original study conducted by the authors. There is no prior publication by any other journal. The corresponding author had full access to all the data in the study and takes responsibility for the integrity of the data and the accuracy of the data analysis as well as the decision to submit for publication. The study was not funded by any institution or body.

\section{Précis}

Inner macular layers should be included together with optic nerve head parameters in optical coherence tomography screening and monitoring of early glaucoma progression.

\section{Bibliography}

1. Spaeth G., et al. "Evaluation of quality of life for patients with glaucoma". American Journal of Ophthalmology 141 (2006): 3-14.

2. Artes PH and Chauhan BC. "Longitudinal changes in the visual field and optic disc in glaucoma". Progress in Retinal and Eye Research 24 (2005): 333-354.

3. Keltner JL., et al. "The association between glaucomatous visual fields and optic nerve head features in the Ocular Hypertension Treatment Study". Ophthalmology 113 (2006): 16031612.

4. Tatham AJ., et al. "Strategies for improving early detection of glaucoma: the combined structure-function index". Clinical Ophthalmology 8 (2014): 611-621.

5. Gardiner SK., et al. "Structural measurements for monitoring change in glaucoma: comparing retinal nerve fiber layer thickness with minimum rim width and area". Investigative Ophthalmology and Visual Science 56 (2015): 6886-6891.

6. Chauhan BC., et al. "Enhanced detection of open angle glaucoma with an anatomically accurate optical coherence tomography-derived neuroretinal rim parameter". Ophthalmology 120 (2013): 535-543.

7. Malik R., et al. "Diagnostic accuracy of optical coherence tomography and scanning laser tomography for identifying glaucoma in myopic eyes". Ophthalmology 123 (2016): 1181-1189.
8. Chauhan BC., et al. "Enhanced detection of open angle glaucoma with an anatomically accurate optical coherence tomography-derived neuroretinal rim parameter". Ophthalmology 120 (2013): 535-543.

9. Gmeiner JM., et al. "Comparison of Bruch's Membrane Opening Minimum Rim Width and Peripapillary Retinal Nerve Fiber Layer Thickness in Early Glaucoma Assessment". Investigative Ophthalmology and Visual Science 57.9 (2016): 575-584.

10. Hood DC. "Improving our understanding, and detection, of glaucomatous damage: an approach based upon optical coherence tomography (OCT)". Progress in Retinal and Eye Research 57 (2017): 46-75.

11. Hood DC., et al. "The nature of macular damage in glaucoma as revealed by averaging optical coherence tomography data". Translational Vision Science and Technology 1 (2012): 3.

12. Hood DC., et al. "Glaucomatous damage of the macula". Progress in Retinal and Eye Research 32 (2013): 1-21.

13. Abe RY., et al. "The impact of location of progressive visual field loss on longitudinal changes in quality of life of patients with glaucoma". Ophthalmology 123 (2016): 552-557.

14. Prager AJ., et al. "Association of glaucoma-related, optical coherence tomography-measured macular damage with vision related quality of life". JAMA Ophthalmology 135 (2017): 783788.

15. Blumberg DM., et al. "Association between undetected 10-2 visual field damage and vision related quality of life in patients with glaucoma". JAMA Ophthalmology 135 (2017): 742-747.

16. Nieves-Moreno M., et al. "Normative database for separate inner retinal layers thickness using spectral domain optical coherence tomography in Caucasian population". PLOS ONE 12.7 (2017): e0180450.

17. Shin HY., et al. "Glaucoma diagnostic ability of ganglion cell-inner plexiform layer thickness differs according to the location of visual field loss". Ophthalmology 121 (2014): 93-99.

18. Kim HJ., et al. "Patterns of glaucoma progression in retinal nerve fiber and macular ganglion cell-inner plexiform layer in spectral-domain optical coherence tomography". Japanese Journal of Ophthalmology 61.4 (2017): 324-333. 
19. Chen MJ., et al. "Diagnostic ability of macular ganglion cell asymmetry in preperimetric glaucoma". BMC Ophthalmology 19 (2019): 12.

20. Traynis I., et al. "Prevalence and nature of early glaucomatous defects in the central 10 degrees of the visual field". JAMA Ophthalmology 132 (2014): 291-297.

21. Hwang YH., et al. "Macular ganglion cell analysis for early detection of glaucoma". Ophthalmology 121 (2014): 1508-1515.

22. Kim YK., et al. "Temporal relation between macular ganglion cell-inner plexiform layer loss and peripapillary retinal nerve fiber layer loss in glaucoma”. Ophthalmology 124 (2017): 1056-1064.

23. Shin JW., et al. "Ganglion cell-inner plexiform layer change detected by optical coherence tomography indicates progression in advanced glaucoma". Ophthalmology 124 (2017): 14661474.

24. Shin JW., et al. "Patterns of progressive ganglion cell-inner plexiform layer thinning in glaucoma detected by OCT". Ophthalmology 125 (2018): 1515-1525.

25. Hou HW., et al. "Integrating macular ganglion cell inner plexiform layer and parapapillary retinal nerve fiber layer measurements to detect glaucoma progression". Ophthalmology 125 (2018): 822-831.

26. Chen MJ., et al. "Diagnostic ability of macular ganglion cell asymmetry in preperimetric glaucoma". BMC Ophthalmology 19 (2019): 12.

27. Leite MT., et al. "Structure-function relationships using the Cirrus spectral domain optical coherence tomograph and standard automated perimetry". Journal of Glaucoma 21 (2012): 49-54.

28. Rao HL., et al. "Retinal nerve fiber layer and macular inner retina measurements by spectral domain optical coherence tomograph in Indian eyes with early glaucoma”. Eye 26 (2012): 133-139.

29. Park K., et al. "Reproducibility of Bruch Membrane Opening Minimum Rim Width Measurements With Spectral Domain Optical Coherence Tomography". Journal of Glaucoma 26 (2017): 1041-1050.

\section{Assets from publication with us}

- Prompt Acknowledgement after receiving the article

- Thorough Double blinded peer review

- Rapid Publication

- Issue of Publication Certificate

- High visibility of your Published work

Website: www.actascientific.com/

Submit Article: www.actascientific.com/submission.php

Email us: editor@actascientific.com

Contact us: +919182824667 